\title{
20-hydroxyeiscosatetraenoic acid may be as a predictor of malignant middle cerebral artery infarction in patients with massive middle cerebral artery infarction
}

\author{
Xingyang $\mathrm{Y}^{1}$, Qiang Zhou ${ }^{2}$, Ting Qing ${ }^{1}$, Bing Ming ${ }^{3}$, Jing Lin ${ }^{2}$, Jie Li ${ }^{1}$ and Jie Lin ${ }^{4 *}$
}

\begin{abstract}
Background: Early identification of massive middle cerebral artery infarction (MCAl) at risk for malignant MCAl ( $\mathrm{m}$-MCAl) may be useful in selecting patients for aggressive therapies. The aim of this study was to determine whether CYP metabolites may help to predict impending m-MCAI.

Methods: This is a prospective, two-center observational study in 256 patients with acute massive MCAl. Plasma levels of 20-hydroxyeicosatetraenoic acid (20-HETE), epoxyeicosatrienoic acids, and dihydroxyeicosatrienoic acids were measured at admission. Brain computed tomography (CT) was performed at admission and repeated between day 3 and 7, or earlier if there was neurological deterioration. The primary outcome was m-MCAI. The m-MCAl was diagnosed when follow-up brain CT detected a more than two-thirds space-occupying MCAl with midline shift, compression of the basal cisterns, and neurological worsening.
\end{abstract}

Results: In total of 256 enrolled patients, 77 (30.1\%) patients developed m-MCAl. Among the 77 patients with m-MCAl, 60 (77.9\%) patients died during 3 months of stroke onset. 20-HETE level on admission was significantly higher in patients with m-MCAl than those without m-MCAl. There was an increase in the risk of m-MCAl with increase of 20-HETE levels. The third and fourth quartiles of 20-HETE levels were independent predictors of m-MCAI (OR: $2.86 ; 95 \% \mathrm{Cl}: 1.16-6.68 ; P=0.025$, and OR: $4.23 ; 95 \% \mathrm{Cl}: 1.35-8.26 ; P=0.002$, respectively).

Conclusions: Incidence of $\mathrm{m}-\mathrm{MCAl}$ was high in patients with massive MCAl and the prognosis of $\mathrm{m}-\mathrm{MCAl}$ is very poor. Elevated plasma 20-HETE may be as a predictor for m-MCAl in acute massive MCAl, and it might useful in clinical practice in therapeutic decision making.

Keywords: 20-hydroxyeicosatetraenoic acid, Brain edema, Malignant middle cerebral artery infarction, Massive middle cerebral artery infarction, Prognosis

*Correspondence: 5337518@qq.com

${ }^{4}$ Department of PET/CT, the First Affiliated Hospital of Wenzhou Medical

University, Wenzhou 325000, Zhejiang, China

Full list of author information is available at the end of the article

\section{Background}

Massive middle cerebral artery infarction (MCAI) accounts for 10 to $15 \%$ of all stroke patients, which usually caused by acute occlusion of internal carotid artery or the proximal middle cerebral artery (MCA) [1, 2], and of these patients, malignant MCAI (m-MCAI) reaches 30 to $50 \%$ [2-4]. The $\mathrm{m}-\mathrm{MCAI}$ is the most devastating form of ischemic stroke, is characterized with 
large supratentorial infarcts and space-occupying brain edema followed by cerebral herniation $[2,5]$. The mortality is approximately $80 \%$ in m-MCAI patients, and those patients who survive experience severe disabilities [6, 7]. Decompressive hemicraniectomy (DHC) is recommended when it is performed within $48 \mathrm{~h}$ after stroke onset in patients with m-MCAI $[8,9]$. Therefore, early identification or prediction of $\mathrm{m}$-MCAI is very essential for timely application of DHC in patients with massive MCAI.

It is noted that not all patients with massive MCAI would develop m-MCAI [2, 4, 10-12]. Therefore, identification of key mechanism involved in m-MCAI and predictor for m-MCAI will be of important significance for the early diagnosis and treatment of m-MCAI among patients with massive MCAI. For the last two decades, prediction of m-MCAI using radiological variables, clinical risk factors, and molecular markers has been thoroughly investigated $[2-4,8,13,14]$. However, the underlying basic mechanisms and predictors for $\mathrm{m}$-MCAI are not completely understood.

The loss of integrity of the endothelial basal lamina and blood-brain barrier (BBB) disruption are believed to be the important cause of edema after focal cerebral ischemia. Arachidonic acid (AA) is a membrane fatty acid that can be metabolized into 20-hydroxyeicosatetraenoic acid (20-HETE) and epoxyeicosatrienoic acids (EETs) by cytochrome P450 (CYP) $\omega$-hydroxylase and CYP epoxygenases, respectively, and EETs are then metabolized to yield less biologically active dihydroxyeicosatrienoic acids (DiHETEs) by soluble epoxide hydrolase (sEH) [15]. 20-HETE and EETs have inflammatory activities [16]. Meanwhile, 20-HETE, as a potent vasoconstrictor, has been shown in the cerebral vasculature of the stroke-prone spontaneously hypertensive rat and contributes to stroke severity [17]. EETs play an important role in neuroprotection and cerebral blood flow regulation after brain injury [18]. Our previous studies demonstrated that CYP metabolite levels were associated with early neurologic deterioration and functional outcome, and may be as a predictor for early neurologic deterioration in acute ischemic stroke $[19,20]$. However, although considerable advances have been made, the association of CYP metabolites with m-MCAI development has not been definitely determined.

In this study, we hypothesized that levels of early CYP metabolites, including 20-HETE, EETs and DiHETEs could predict m-MCAI. Therefore, we conducted a prospective, two-center observational study that aimed to explore potential CYP metabolites involved in the $\mathrm{m}-\mathrm{MCAI}$ and to elucidate their possible mechanisms in m-MCAI development.

\section{Materials and methods}

\section{Study population}

This study was conducted in People's Hospital of Deyang City, the First Affiliated Hospital of Wenzhou Medical University and the Third Affiliated Hospital of Wenzhou Medical University. The study protocol was approved by the Ethics Committee at the participating hospitals. Written informed consent was obtained from each patient prior to study enrollment.

Between October 2011 and September 2014, we consecutively registered 1542 patients who had suffered their first-ever stroke and were admitted to the participating hospitals. Data were recorded at the time of assessment using a standardized structured form. Detailed methods for data collection of the patients were described in our previous articles $[14,21]$. In this study, we enrolled the patients who were suffered from massive MCAI and admitted to the participating hospitals within $48 \mathrm{~h}$ of their index stroke onset. Massive MCAI was defined as $>50 \%$ of the MCA territory on early cranial computed tomography $(\mathrm{CT})$ or magnetic resonance imaging (MRI) scans, with or without the involvement of the adjacent territories $[14,22]$. The patients with incomplete hospital records or missing imaging, preexisting score of more than 2 on the modified Rankin scale (mRS), participating in other clinical trials or receiving thrombectomy, and unwilling to participate in this study were excluded. According to aforementioned inclusion criteria and exclusion criteria, a total of 256 patients with massive MCA infarction were enrolled, the detailed data cleaning procedure was presented in Fig. 1.

All enrolled patients had an initial brain CT scan at admission. Early signs of cerebral infarction on CT included: (1) the presence of focal hypodensity consistent with the clinical findings,obscuration of the lenticular nucleus or the cortex; (2) mass effect was determined by grading hemispheric swelling [23]: effacement of the cortical sulci (grade I), ventricular asymmetry grade II), shifting of the structures of the median line (grade III) [23]. For the extent of MCAI, Alberta Stroke Program Early CT Score (ASPECTS) was evaluated in the first CT examination [24]. A second CT or MRI was performed between 3 days and 7 days of hospitalization to measure the infarct volume. The infarct volume was determined using the Coniglobus formula $0.5 \times a \times b \times c$ (where $a$ and $b$ are the largest perpendicular diameters measured and $c$ is the sum of slices multiplied with thickness on CT or diffusion-weighted imaging on MRI). An additional CT scan was performed to determine brain edema or hemorrhagic transformation whenever patients had neurological deterioration. Assessment of brain CT or MRI was conducted by a neuroradiologist who was blinded to clinical picture. 


\section{We registered 1542 patients with ischemic stroke who were admitted within 48 hour of the onset of stroke}

According to cranial computed tomography or magnetic resonance imaging
scans, 256 patients were massive middle cerebral artery infarction (MCAI).
Plasma CYP450 metabolite levels (20-HETE, EETs and DiHETEs ) were
measured in the 256 patients with massive MCAI at adimission

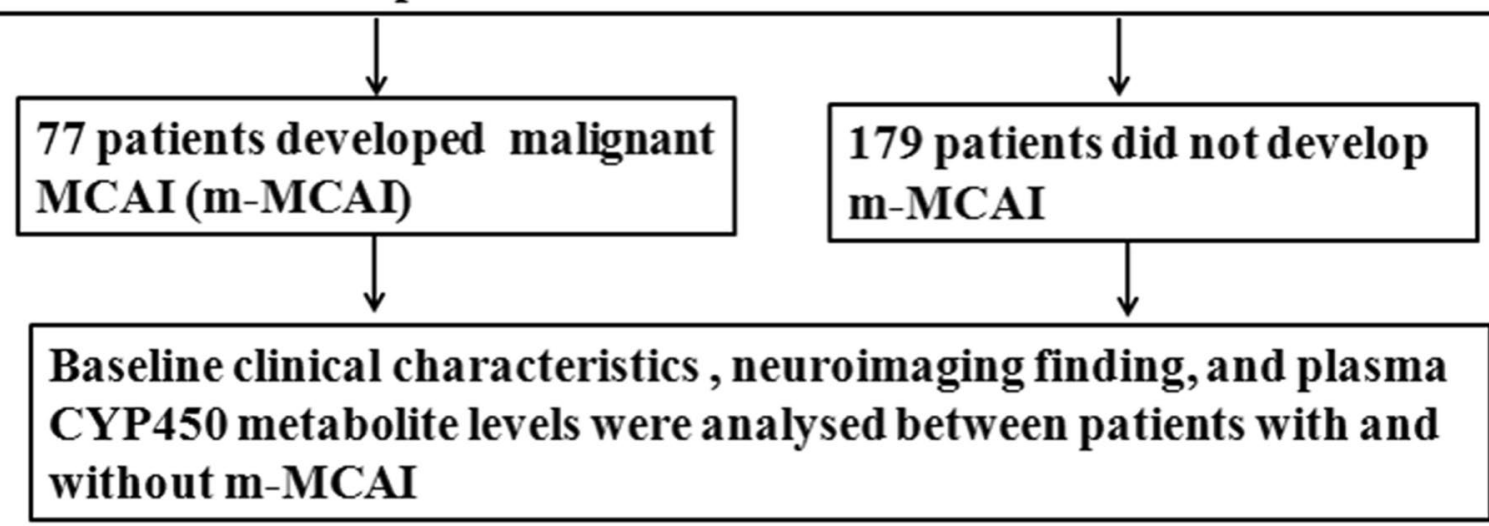

Fig. 1 Flowchart in this study

For each patient, stroke severity was assessed using National Institutes of Health Stroke Scale (NIHSS) by a member of stroke team on admission, and subsequently on a daily during period of hospitalization. Age, sex, onset to admission time, baseline systolic and diastolic blood pressure were recorded. Vascular risk factors were investigated. Serum glucose, triglycerides (TG), total plasma cholesterol (TC), and low-density lipoprotein cholesterol (LDL-C) were measured. Dyslipidemia was defined as TG $>180 \mathrm{mg} / \mathrm{dL}, \mathrm{TC}>200 \mathrm{mg} / \mathrm{dL}$ or use of lipid-lowering medication [19]. Stroke subtypes were classified as large-artery atherosclerosis, cardioembolism, stroke of other determined etiology, and stroke of undetermined etiology according to the subtype classification criteria [25]. All enrolled patients received standard therapies based on standard guidelines [9].

Measurement of plasma 20-HETE, EETs and DiHETEs levels Whole blood $(4 \mathrm{ml})$ was drawn from each patient on admission. Plasma was isolated following centrifugation and samples were stored at $-80^{\circ} \mathrm{C}$ until analysis. Total plasma EETs and DiHETEs levels were measured using a stable isotope dilution GC/MS following base hydrolysis and separation on high performance liquid chromatography (HPLC), and plasma 20-HETE level was analyzed using a stable isotope dilution gas chromatography/mass spectrometer (GC/MS), as described in our previous studies $[19,20]$.

Assessment of $\mathrm{m}$-MCAl and clinical outcome

The primary outcome of this study was m-MCAI. The $\mathrm{m}$-MCAI was diagnosed according to the following criteria [13, 26]: massive MCAI showed on follow-up CT including more than two-thirds space-occupying MCAI with midline shift and compression of the basal cisterns, and further consciousness status declined at least 1 point of consciousness item described in the NIHSS compared with the baseline consciousness status on admission, or deterioration of neurological status with clinical signs of uncal herniation and mass effect leading to early death or DHC.

All patients were followed up at 3 months after stroke by a certified stroke team member using questionnaires via telephone interview. The secondary outcome was vascular death and modified Rankin Scale (mRS) at 3 months. Vascular death was defined as vascular mortality due to coronary heart disease, ischemic stroke, or other vascular causes. A favorable outcome was considered as $\mathrm{mRS} \leq 2$ points, while $\mathrm{mRS}>2$ points was defined as a unfavorable outcome [14, 19-21]. 


\section{Statistical analysis}

Previous studies have shown prevalence of $\mathrm{m}$-MCAI is approximately $30-50 \%$ in patients with massive MCAI [2-4]. According to this estimate, we expected a minimum sample size requirement of 250 patients with massive MCAI for determining the difference in prevalence of m-MCAI within $\pm 15 \%$ with $95 \%$ confidence intervals (CI) $[20,27]$.

The results are expressed as percentages for categorical variables, and continuous variables are expressed as mean \pm Standard Deviation. Baseline and clinical characteristics were compared using $X^{2}$ test or Fisher exact test (categorical variables) and the Student $t$ test (continuous variables) between patients with and without m-MCAI. We calculated 20-HETE by quartiles of increasing levels to evaluate for possible threshold effects for m-MCAI.

Multiple logistic regression analysis was used to assess the possible contributing factors for m-MCAI using variables with $P$ values $<0.05$ in univariate analysis, and reported as odds ratio (OR) with the 95\% confidence intervals (CI). Furthermore, Cox proportional hazard model was performed to account for the probability of m-MCAI according to the 20-HETE levels levels, and reported using Kaplan-Maier curve.

All statistical analyses were performed using SPSS 16.0 (SPSS Inc., Chicago, IL, USA). All tests were two sided, and the threshold level of $P<0.05$ was defined as statistical significance.

\section{Results}

We prospectively registered 1542 patients with ischemic stroke who were admitted within $48 \mathrm{~h}$ of the onset of stroke between October 2011 and September 2014. Among the 1542 patients, 256 patients were massive MCAI. Seventy-seven of the 256 patients (30.1\%) developed m-MCAI (60 of 77 patients [77.9\%] occurred in the first $72 \mathrm{~h}$, all occurred within 7 days after admission), 179 patients $(69.9 \%)$ did not experience m-MCAI. Baseline clinical characteristics in patients with and without m-MCAI were presented in Table 1. Compared with

Table 1 Baseline clinical characteristics in patients with and without m-MCAI

\begin{tabular}{|c|c|c|c|}
\hline Characteristics & $\begin{array}{l}\text { Patients with m-MCAI } \\
(n=77)\end{array}$ & $\begin{array}{l}\text { Patients without m-MCAI } \\
(n=179)\end{array}$ & $P$ value \\
\hline Age (years) & $58.9 \pm 14.2$ & $66.7 \pm 15.5$ & $<0.001$ \\
\hline Men $(n, \%)$ & $36(46.8)$ & $87(48.6)$ & 0.786 \\
\hline$>60$ y of age $(n, \%)$ & $32(41.6)$ & $108(60.3)$ & $<0.001$ \\
\hline Hypertension (n, \%) & $35(45.5)$ & $94(52.5)$ & 0.301 \\
\hline Diabetes mellitus (n, \%) & $16(20.8)$ & $37(20.7)$ & 0.987 \\
\hline Atrial fibrillation (n, \%) & $45(58.4)$ & $66(36.9)$ & 0.002 \\
\hline Current smoker (n, \%) & $19(24.7)$ & $39(21.8)$ & 0.637 \\
\hline Dyslipidemia (n, \%) & $12(15.6)$ & 35 (19.6) & 0.482 \\
\hline Plasma glucose (mmol/L) & $7.7 \pm 3.2$ & $7.9 \pm 3.5$ & 0.652 \\
\hline Onset to admission time (h) & $21.3 \pm 8.9$ & $23.2 \pm 10.2$ & 0.151 \\
\hline NIHSS score on admission & $16.1 \pm 7.3$ & $14.9 \pm 7.9$ & 0.147 \\
\hline SBP on admission ( $\mathrm{mm} \mathrm{Hg}$ ) & $146.7 \pm 26.4$ & $144.7 \pm 25.2$ & 0.578 \\
\hline DBP on admission (mm Hg) & $84.8 \pm 14.7$ & $85.3 \pm 15.6$ & 0.712 \\
\hline Temperature on admission, ${ }^{\circ} \mathrm{C}$ & $36.9 \pm 1.5$ & $36.7 \pm 1.7$ & 0.349 \\
\hline Left MCA infarction (n, \%) & 49 (63.6) & $78(43.6)$ & 0.004 \\
\hline Etiology (n, \%) & & & 0.014 \\
\hline Large-artery atherosclerosis & $16(20.8)$ & $43(24.0)$ & \\
\hline Cardioembolism & $49(63.6)$ & $74(41.3)$ & \\
\hline Other determined etiology & $2(2.6)$ & $12(6.7)$ & \\
\hline Undetermined etiology & $10(13.0)$ & $50(27.9)$ & \\
\hline Mechanical ventilation (n, \%) & $27(35.1)$ & $3(1.7)$ & $<0.001$ \\
\hline Decompressive craniectomy (n, \%) & $21(27.2)$ & $3(1.7)$ & $<0.001$ \\
\hline \multicolumn{4}{|l|}{ Outcome $(n, \%)$} \\
\hline Early neurological deterioration & $77(100.0)$ & $47(26.3)$ & $<0.001$ \\
\hline Mortality during 3 months & $60(77.9)$ & $75(41.9)$ & $<0.001$ \\
\hline mRS $>2$ points at 3 months & $74(96.1)$ & $128(71.5)$ & $<0.001$ \\
\hline
\end{tabular}

m-MCAI malignant middle cerebral artery infarction, MCA middle cerebral artery; NIHSS National Institutes of Health Stroke Scale, SBP systolic blood pressure, DBP diastolic blood pressure, $t P A$ tissue plasminogen activator, $m R S$ modified Rankin Scale 
patients without m-MCAI, younger age, atrial fibrillation, left MCAI, and subtype of cardio-embolism were significantly more frequent in patients with m-MCAI. For in-hospital management, patients with m-MCAI more frequently used mechanical ventilation $(35.1 \%$ vs. $1.7 \%$, $P<0.001)$ and decompressive surgery $(27.2 \%$ vs. $1.7 \%$, $P<0.001$ ) than patients without m-MCAI (Table 1 ).

Unfavorable outcome $(\mathrm{mRS}>2)$ was more frequent in patients with m-MCAI than those without m-MCAI. Sixty of patients $(77.9 \%)$ with m-MCAI died within 3 months after stroke, and only 3 (3.9\%) of the survivors were independent $(\mathrm{mRS} \leq 2)$ at 3 months. Among the patients without m-MCAI, 75 (41.9\%) patients were dead during 3 months, and 51 (25.5\%) were independent at 3 months (Table 1 ).

Early signs of cerebral infarction in the first CT scan were detected more frequently in patients who later developed m-MCAI than those did not experience m-MCAI ( $89.6 \%$ vs. $77.8 \% ; P=0.045$, Table 2 ). Severe mass effect (grade II and III) and extent of MCAI (ASPECTS) on CT scan at admission, and final infarct volume $>145 \mathrm{~cm}^{3}$ at second CT scan were significantly more frequent in m-MCAI group than non m-MCAI group (Table 2).

The mean 20-HETE level was $1924.6 \pm 194.8 \mathrm{pmol} / \mathrm{L}$ in patients with $\mathrm{m}-\mathrm{MCAI}$, and $1698.7 \pm 173.5$ in patients without $\mathrm{m}-\mathrm{MCAI}$ on admission $(P<0.001$, Table 2$)$. There were no significant differences of EETs and DiHETEs levels between two groups $(P>0.05$, Table 2). The mean 20 -HETE level was $1789 \pm 174.6 \mathrm{pmol} / \mathrm{L}$ in 256 patients with massive MCAI, with quartile levels as follows: 826 to $1456 \mathrm{pmol} / \mathrm{L}$ (first quartile, $n=48$ ); 1457 to $1768 \mathrm{pmol} / \mathrm{L}$ (second quartile $n=78$ ); 1769 to $2024 \mathrm{pmol} / \mathrm{L}$ (third quartile, $n=76$ ); and 2025 to $2737 \mathrm{pmol} / \mathrm{L}$ (fourth quartile, $n=54)$. The incidence of m-MCAI increased with increasing quartile levels of 20-HETE (0\% [0/48], $14.1 \%[11 / 78], 35.5 \%$ [27/76], and $72.2 \%$ [39/54] in patients from first quartile to fourth quartile, respectively, $P<0.001)$. The median time of developed m-MCAI in patients with massive MCAI was 4 days after admission for low 20-HETE levels, $32 \mathrm{~h}$ for intermediated 20-HETE levels, and $18 \mathrm{~h}$ for high 20-HETE levels.

20-HETE levels and final infarct volume $>145 \mathrm{~cm}^{3}$ were found to be independent predictors of m-MCAI after adjusting the covariates (Table 3). The odds ratio for $\mathrm{m}$-MCAI increased with increase in quartiles of 20-HETE level, using the first quartile as the reference,

Table 3 Predictors of $\mathrm{m}-\mathrm{MCAl}$ and odds ratio according to 20-HETE quartiles

\begin{tabular}{llll}
\hline Factor & OR & $\mathbf{9 5 \% ~ C l}$ & P value \\
\hline $\begin{array}{l}\text { 20-HETE Quartile, pmol/L } \\
\quad \text { Quartile 1 (reference) }\end{array}$ & & & \\
$\quad$ Quartile 2 & 1.23 & $0.54-2.96$ & 0.425 \\
$\quad$ Quartile 3 & 2.86 & $1.16-6.68$ & 0.025 \\
$\quad$ Quartile 4 & 4.23 & $1.35-8.26$ & 0.002 \\
Age > 60 y & 0.94 & $0.83-1.64$ & 0.532 \\
Atrial fibrillation & 1.33 & $0.75-2.04$ & 0.342 \\
Left MCA infarction & 1.42 & $0.96-3.87$ & 0.126 \\
Cardioembolism & 1.33 & $0.97-3.58$ & 0.113 \\
Early signs of CT & 1.21 & $0.89-2.21$ & 0.325 \\
Mass effect (Grade II and III) & 1.38 & $0.98-4.36$ & 0.063 \\
ASPECTS $\leq 4$ & 1.22 & $0.99-3.96$ & 0.083 \\
Final infarct volume $>145 \mathrm{~cm}^{3}$ & 1.67 & $1.46-6.25$ & 0.026
\end{tabular}

$m-M C A /$ malignant middle cerebral artery infarction, $M C A$ middle cerebral artery; HETE hydroxyeicosatetraenoic acid, $\mathrm{Cl}$ confidence intervals, $O R$ odds ratio, $C T$ computed tomography, ASPECTS Alberta Stroke Program Early CT Score

Table 2 Neuroimaging finding and plasma CYP450 metabolite levels on admission in patients with and without m-MCAI

\begin{tabular}{|c|c|c|c|}
\hline Factor & $\begin{array}{l}\text { Patients with m-MCAI } \\
(n=77)\end{array}$ & $\begin{array}{l}\text { Patients without m-MCAI } \\
(n=179)\end{array}$ & $P$ value \\
\hline Early signs at the first CT scan (n, \%) & $69(89.6)$ & $141(78.8)$ & 0.045 \\
\hline Hypodensity & $59(76.6)$ & $139(77.7)$ & 0.863 \\
\hline Mass effect & $56(72.7)$ & $91(50.8)$ & $<0.001$ \\
\hline Grade I & $15(19.5)$ & $80(44.7)$ & \\
\hline Grade II & $27(35.1)$ & $11(6.1)$ & \\
\hline Grade III & $14(18.2)$ & $0(0.0)$ & \\
\hline ASPECTS & $4(3-6)$ & $7(6-10)$ & 0.036 \\
\hline Infarct volume $>145 \mathrm{~cm}^{3}$ at second CT scan $(\mathrm{n}, \%)$ & $71(92.2)$ & $18(10.1)$ & $<0.001$ \\
\hline 20-HETE at admission (pmol/L) & $1924.6 \pm 194.8$ & $1698.7 \pm 173.5$ & $<0.001$ \\
\hline EETs at admission (nmol/l) & $66.7 \pm 17.6$ & $70.4 \pm 18.9$ & 0.123 \\
\hline DiHETEs at admission (nmol/l) & $80.2 \pm 19.1$ & $75.8 \pm 17.6$ & 0.089 \\
\hline
\end{tabular}


the third and fourth quartiles of 20-HETE levels were found to be independent predictors of m-MCAI (OR: 2.86; $95 \%$ CI: $1.16-6.68 ; P=0.025$, and OR: 4.23 ; $95 \%$ CI: $1.35-8.26 ; P=0.002$, respectively, Table 3$)$. Cox proportional hazard curve is showed in the Fig. 2, there was an increase in the risk of m-MCAI with higher level of 20-HETE (HR: 3.82, 95\% CI: $1.54-9.23, P=0.001$ (logrank test).

\section{Discussion}

The present results showed that the incidence of $\mathrm{m}$-MCAI was very high in patients with massive MCAI. 20-HETE level was independent predictor of m-MCAI, the odds ratio for $\mathrm{m}$-MCAI increased with increase in quartiles of 20-HETE levels, this may be of use in therapeutic decision making and the timing of DHC [28].

In recent decades, clinical risk factors, neuroimaging variables, and molecular markers have been thoroughly investigated for prediction of m-MCAI [2-4, 8, 13, 14]. However, the sensibility and specificity of these markers for prediction of m-MCAI are insufficient. Some studies revealed that the sensitivity of brain $\mathrm{CT}$ scan was high, while the specificity was low for identifying m-MCAI $[23,29]$. In present study, we found that prevalence of early signs of cerebral infarction and mass effect (grade II and grade III) was more prevalent, and ASPECTS was significantly lower on the first CT scan in patients who later developed m-MCAI than those did not experience m-MCAI. However, these neuroimaging variables were not independent predictor of m-MCAI. Our results were in accordance with other previous studies [5, 8, 23, 29], indicating that clinical factors were not sufficient to identify patients with impending malignant brain edema and $\mathrm{m}-\mathrm{MCAI}[2,14]$. In recent study, promising results have been obtained from neuroimaging tests such as singlephoton emission CT and diffusion-weighted MRI in the prediction of m-MCAI [30]. Although these techniques are most reliable predictor for $\mathrm{m}-\mathrm{MCAI}$ quickly and accurately, they are unable to directly predict the development of massive brain edema.

The underlying basic mechanisms of m-MCAI are not completely understood. The loss of integrity of the endothelial basal lamina and BBB disruption play key roles in pathophysiological mechanisms of malignant edema formation after massive MCAI. In this study, we found that elevated plasma 20-HETE may be as an independent predictor for $\mathrm{m}$-MCAI in patients with acute massive MCAI, and this is the first to identify a positive relationship between 20-HETE levels and m-MCAI.

20-HETE, as a potent vasoconstrictor, is associated with cerebral ischemia injury, cerebral edema, and unfavorable outcomes after subarachnoid hemorrhage [31]. The increase of vascular permeability and subsequent extravasation of serum components is one of principal causes in development of brain edema after ischemic stroke. 20-HETE can regulate cerebral vascular tone, constrict cerebral arteries, and increase vascular permeability by activating intracellular protein kinase $\mathrm{C}$ signaling

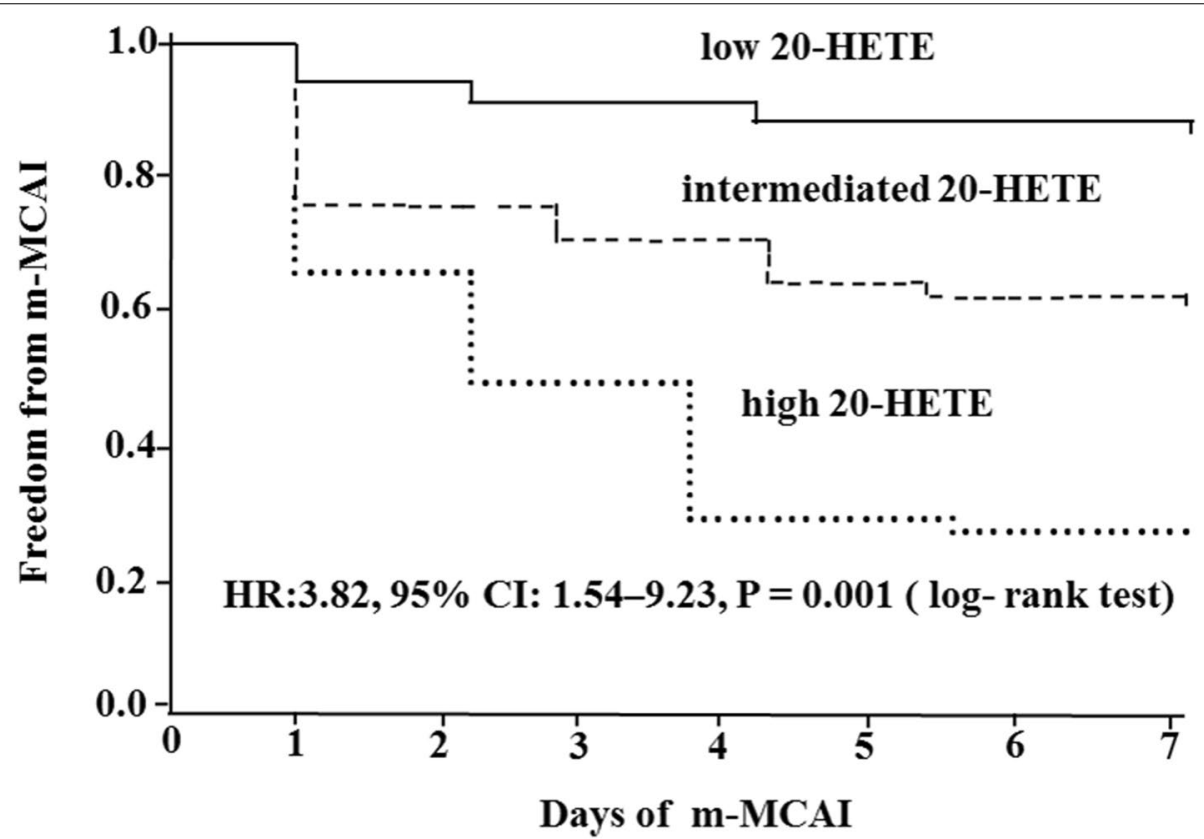

Fig. 2 Probability of survival free of m-MCAl. Kaplan-Maier analysis of cumulative freedom from m-MCAl associated with HETE. m-MCAI indicates malignant middle cerebral artery infarction; HETE indicates hydroxyeicosatetraenoic acid 
pathway [32], which is involved in apoptosis or cell death [33]. Neuronal apoptosis is an important mechanism of brain ischemic damage in animal experiments [34], and is associated with mortality and poor functional prognosis after ischemic stroke [35]. 20-HETE may also injure vascular smooth muscle cells and endothelial cells by inhibition of $\mathrm{Na}^{+}, \mathrm{K}^{+}-$ATPase activity and large-conductance $\mathrm{Ca}^{2+}$ - sensitive $\mathrm{K}^{+}$channel, and increase of $\mathrm{Ca}^{2+}$ influx via $\mathrm{L}$-type $\mathrm{Ca}^{+}{ }^{+}$channels [36]. In addition, 20-HETE promotes formation of oxygen radicals, which may cause endothelial injury $[37,38]$. Inhibitor of 20-HETE can reverse the decrease of cerebral blood flow following subarachnoid hemorrhage and reduce infarct size after transient cerebral ischemia in animal experiments $[39,40]$. All of these previous studies suggest a potential molecular mechanism of 20-HETE might lead to m-MCAI after acute massive MCAI.

Our previous study showed that low EETs levels and high DiHETEs levels were associated with early neurological deterioration in minor ischemic stroke (NIHSS score $\leq 3)[20]$. However, we did not find such association for m-MCAI in this study. In present study, we enrolled patients with acute massive MCAI, NIHSS scores on admission were higher, standard deviations of NIHSS, EETs levels and DiHETEs levels were bigger than patients with minor ischemic stroke. Furthermore, the small samples were also another reason of no association between low EETs levels and m-MCAI. Thus, larger samples studies are necessary to validate our present findings in future.

Despite our findings are interesting, several limitations of this study should be noted. First, this was a two-center study, the samples were small. Therefore, our current findings should be validated in larger samples, multicenter studies. Second, plasma CYP metabolite levels may dynamic changes after ischemic stroke. Blood samples for CYP metabolites were drawn from each patient at admission. We did not measure plasma CYP metabolite levels during follow-up in non-surviving and surviving patients. Third, $\mathrm{m}$-MCAI could be better detected if brain CT scan were consistently undertaken earlier, or repeated every $48 \mathrm{~h}$. However, brain CT scan was performed at admission and repeated between 3 days and 7 days of hospitalization or when patients had neurological deterioration in this study. Therefore, further well designed studies are needed to confirm our current findings.

\section{Conclusion}

The incidence of m-MCAI was very high in patients with massive MCAI, the prognosis of $\mathrm{m}-\mathrm{MCAI}$ is very poor. Elevated plasma 20 -HETE may be as a predictor for m-MCAI.
Our findings may be useful in clinical practice in therapeutic decision making and the timing of DHC.

\begin{abstract}
Abbreviations
MCAl: Massive middle cerebral artery infarction; m-MCAl: Malignant MCAI; DHC: Decompressive hemicraniectomy; 20-HETE: 20-hydroxyeicosatetraenoic acid; CT: Computed tomography; ASPECTS: Alberta Stroke Program Early CT Score; BBB: Blood-brain barrier; CYP: Cytochrome P450; EETs: Epoxyeicosatrienoic acids; sEH: Soluble epoxide hydrolase; MRI: Magnetic resonance imaging; mRS: Modified Rankin scale; TG: Triglycerides; TC: Total plasma cholesterol; LDLC: Low-density lipoprotein cholesterol; Cl: Confidence intervals; OR: Odds ratio.
\end{abstract}

\section{Acknowledgments}

None.

\section{Authors' contributions}

XY participated in the design of the study and drafted the manuscript. QZ participated in the design of the study and statistical analysis. JL1 and BM participated in the design of the study, brain computed tomography and magnetic resonance imaging. JL2 and TQ wrote the main manuscript text. QZ and JL3 participated to evaluate patients. All authors reviewed and approved the final manuscript.

\section{Funding}

This study was supported in part by grants from the Sichuan Science and Technology Agency Research Foundation (Grant No.2018JY0164), the Scientific Research Foundation of Sichuan Provincial Health Department (Grant No. 16ZD046). The funding body did not participate in the design of the study;

collection, analysis, and interpretation of data; and in writing the manuscript.

\section{Availability of data and materials}

The datasets used and/or analysed during the current study are available from the corresponding author on reasonable request.

\section{Declarations}

\section{Ethics approval and consent to participate}

The study protocol was submitted to and approved by the Ethics Committee of the People's Hospital of Deyang City, the Affiliated Hospital of Southwest Medical University, and Suining Central Hospital. All procedures performed in studies involving human participants were in accordance with the ethical standards of the institutional and national research committee and with the 1964 Helsinki declaration and its later amendments or comparable ethical standards. Written informed consent was obtained from all individual participants included in the study.

\section{Consent for publication \\ Not applicable.}

\section{Competing interests}

The authors declare that they have no competing interests.

\section{Author details}

'Department of Neurology, People's Hospital of Deyang City, Deyang 618000, Sichuan, China. ${ }^{2}$ Department of Neurology, the Third Affiliated Hospital of Wenzhou Medical University, Wenzhou 325200, Zhejiang, China. ${ }^{3}$ Department of Radiology, People's Hospital of Deyang City, Deyang 618000, Sichuan, China. ${ }^{4}$ Department of PET/CT, the First Affiliated Hospital of Wenzhou Medical University, Wenzhou 325000, Zhejiang, China.

Received: 30 December 2020 Accepted: 20 October 2021

Published online: 09 November 2021

\section{References}

1. Moulin DE, Lo R, Chiang J, Barnett HJM. Prognosis in middle cerebral artery occlusion. Stroke. 1985;16:282-4. 
2. Huttner HB, Schwab S. Malignant middle cerebral artery infarction: clinical characteristics, treatment strategies, and future perspectives. Lancet Neurol. 2009;8:949-58.

3. Kasner SE, Demchuk AM, Berrouschot J, Schmutzhard E, Harms L, Verro $P$, et al. Predictors of fatal brain edema in massive hemispheric ischemic stroke. Stroke. 2001;32:2117-23.

4. Dohmen C, Bosche B, Graf R, Staub F, Kracht L, Sobesky J, et al. Prediction of malignant course in MCA infarction by PET and microdialysis. Stroke. 2003:34:2152-8.

5. Berrouschot J, Sterker M, Bettin S, Köster J, Schneider D. Mortality of space-occupying ('malignant') middle cerebral artery infarction under conservative intensive care. Intensive Care Med. 1998;24:620-3.

6. Hacke W, Schwab S, Horn M, Spranger M, De Georgia M, von Kummer R. "Malignant" middle cerebral artery territory infarction: clinical course and prognostic signs. Arch Neurol. 1996;53:309-15.

7. Steiner T, Ringleb P, Hacke W. Treatment options for large hemispheric stroke. Neurology. 2001;57(Suppl.2):S61-8.

8. Minnerup J, Wersching H, Ringelstein EB, Heindel W, Niederstadt T, Schilling $M$, et al. Prediction of malignant middle cerebral artery infarction using computed tomography-based intracranial volume reserve measurements. Stroke. 2011;42:3403-9.

9. Powers WJ, Rabinstein AA, Ackerson T, Adeoye OM, Bambakidis NC, Becker K, et al. 2018 guidelines for the early management of patients with acute ischemic stroke: a guideline for healthcare professionals from the American Heart Association/American Stroke Association. Stroke. 2018;49:e46-e110.

10. Neugebauer $H$, Jüttler $E$. Hemicraniectomy for malignant middle cerebral artery infarction: current status and future directions. Int J Stroke. 2014;9:460-7

11. Frank Jl. Large hemispheric infarction, deterioration, and intracranial pressure. Neurology. 1995:45:1286-90.

12. Qureshi Al, Suarez Jl, Yahia AM, Mohammad Y, Uzun G, Suri MF, et al. Timing of neurologic deterioration in massive middle cerebral artery infarction: a multicenter review. Crit Care Med. 2003;31:272-7.

13. Zhou Z, Zhang J, Li X, Xia C, Han Y, Chen H. Protein microarray analysis identifies key cytokines associated with malignant middle cerebralartery infarction. Brain Behav. 2017;7:e00746.

14. Li J, Zhang P, Wu S, Yi X, Wang C, Liu M. Factors associated with favourable outcome in large hemispheric infarctions. BMC Neurol. 2018;18:152.

15. Zeldin DC. Epoxygenase pathways of arachidonic acid metabolism. J Biol Chem. 2001;276:36059-62.

16. Calder PC. Polyunsaturated fatty acids and inflammatory processes: new twists in an old tale. Biochimie. 2009;91:791-5.

17. Dunn KM, Renic M, Flasch AK, Harder DR, Falck J, Roman RJ. Elevated production of 20-HETE in the cerebral vasculature contributes to severity of ischemic stroke and oxidative stress in spontaneously hypertensive rats. Am J Physiol Heart Circ Physiol. 2008;295:H2455-65.

18. Imig JD, Simpkins AN, Renic M, Harder DR. Cytochrome P450 eicosanoids and cerebral vascular function. Exp Rev Mol Med. 2011;13:e7.

19. Yi X, Han Z, Zhou Q, Lin J, Liu P. 20-hydroxyeicosatetraenoic acid as a predictor of neurological deterioration in acute minor ischemic stroke. Stroke. 2016;47:3045-7.

20. Yi X, Lin J, Li J, Zhou Q, Han Z. Epoxyeicosatrienoic Acids are Mediated by EPHX2 Variants and may be a Predictor of Early Neurological Deterioration in Acute Minor Ischemic Stroke. J Atheroscler Thromb. 2017;24:1258-66

21. Yi X, Lin J, Wang C, Zhang B, Chi W. Low-molecular-weight heparin is more effective than aspirin in preventing early neurologic deterioration and improving sixmonth outcome. J Stroke Cerebrovasc Dis. 2014:23:1537-44.

22. Uhl E, Kreth FW, Elias B, Goldammer A, Hempelmann RG, Liefner M, et al. Outcome and prognostic factors of hemicraniectomy for space occupying cerebral infarction. J Neurol Neurosurg Psychiatry. 2004;75:270-4.

23. Serena J, Blanco M, Castellanos M, Silva Y, Vivancos J, Moro MA, et al. The prediction of malignant cerebral infarction by molecular brain barrier disruption markers. Stroke. 2005:36:1921-6.
24. Pexman $\mathrm{H}$, Barber PA, Hill MD, Sevick RJ, Demchuk AM, Hudon ME et al. Use of the Alberta stroke program early CT score (ASPECTS) for assessing CT scans in patients with acute stroke. AJNR Am J Neuroradiol. 2001;22:1534-42.

25. Han SW, Kim SH, Lee JY, Chu CK, Yang JH, Shin HY, et al. A new subtype classification of ischemic stroke based on treatment and etiologic mechanism. Eur Neurol. 2007:57:96-102.

26. Schwab S, Steiner T, Aschoff A, Schwarz S, Steiner HH, Jansen O, et al. Early hemicraniectomy in patients with complete middle cerebral artery infarction. Stroke. 1998;29:1888-93.

27. Lwanga SK, Lemeshow S. Sample size determination in health studies: a practical manual. Geneva: World Health Organization; 1991.

28. Kilic M, Ertem DH, Ozdemir B. Timing of decompressive craniectomy for malignant middle cerebral artery infarction: a single-center analysis. Medicina (Kaunas). 2019;55(2). https://doi.org/10.3390/medicina55 020031.

29. von Kummer R, Meyding-Lamade U, Forsting M, Rosin L, Rieke K, Hacke W, et al. Sensitivity and prognostic value of early CT in occlusion of the middle cerebral artery trunk. AJNR Am J Neuroradiol. 1994;15:9-15.

30. Pallesen LP, Barlinn K, Puetz V. Role of decompressive craniectomy in ischemic stroke. Front Neurol. 2019;9:1119. https://doi.org/10.3389/fneur. 2018.01119 eCollection 2018.

31. Donnelly MK, Crago EA, Conley YP, Balzer JR, Ren D, Ducruet AF, et al. 20-HETE is associated with unfavorable outcomes in subarachnoid hemorrhage patients. J Cereb Blood Flow Metab. 2015;35:1515-22.

32. Lange A, Gebremedhin D, Narayanan J, Harder D. 20-Hydroxyeicosatetraenoic acid-induced vasoconstriction and inhibition of potassium current in cerebral vascular smooth muscle is dependent on activation of protein kinase C. J Biol Chem. 1997;272:27345-52.

33. Randriamboavonjy V, Busse R, Fleming I. 20-HETE induced contraction of small coronary arteries depends on the activation of rho-kinase. Hypertension. 2003;41:801-6.

34. Sairanen T, Karjalainen-Lindsberg ML, Paetau A, ljäs P, Lindsberg PJ. Apoptosis dominant in the periinfarct area of human ischaemic stroke--a possible target of antiapoptotic treatments. Brain. 2006;129:189-99.

35. Gomez-Sanchez JC, Delgado-Esteban M, Rodriguez-Hernandez I, Sobrino T, Perez De La Ossa N, Reverte S, et al. The human Tp53 Arg72Pro polymorphism explains different functional prognosis in stroke. J Exp Med. 2011;208:429-37

36. Cheng J, Ou JS, Singh H, Falck JR, Narsimhaswamy D, Pritchard KA Jr, et al. 20-Hydroxyeicosatetraenoic acid causes endothelial dysfunction via eNOS uncoupling. Am J Physiol Heart Circ Physiol. 2008;294:H1018-26.

37. Guo AM, Arbab AS, Falck JR, Chen P, Edwards PA, Roman RJ, et al. Activation of vascular endothelial growth factor through reactive oxygen species mediates 20-hydroxyeicosatetraenoic acid-induced endothelial cell proliferation. J Pharmacol Exp Ther. 2007;321:18-27.

38. Singh H, Cheng J, Deng H, Kemp R, Ishizuka T, Nasjletti A, et al. Vascular cytochrome P450 4A expression and 20-hydroxyeicosatetraenoic acid synthesis contribute to endothelial dysfunction in androgen-induced hypertension. Hypertension. 2007;50:123-9.

39. Takeuchi K, Renic M, Bohman QC, Harder DR, Miyata N, Roman RJ. Reversal of delayed vasospasm by an inhibitor of the synthesis of 20-HETE. Am J Physiol Heart Circ Physiol. 2005;289:H2203-11.

40. Tanaka Y, Omura T, Fukasawa M, Horiuchi N, Miyata N, Minagawa T, et al. Continuous inhibition of 20-HETE synthesis by TS-011 improves neurological and functional outcomes after transient focal cerebral ischemia in rats. Neurosci Res. 2007;59:475-80.

\section{Publisher's Note}

Springer Nature remains neutral with regard to jurisdictional claims in published maps and institutional affiliations. 